Pulmonary tumour embolism is a rare condition without specific symptoms or pathognomonic features. Pulmonary tumour embolism can occur as the first manifestation of cancer, but because of diagnostic difficulties, it is often wrongly recognised as a more common cardiopulmonary disease. We present a case of a 46 -year-old Caucasian male with no prior malignancy diagnosis, admitted because of progressing dyspnoea and cough. Based on radiological and clinical presentations, sarcoidosis, silicosis and lymphangitic carcinomatosis were considered in the differential diagnosis. Histopathological analysis of lung biopsy revealed that multiple emboli of atypical epithelial cells found in the pulmonary vessels were of gastrointestinal origin. Further pathological examination of the gastric biopsy led to the final diagnosis of the signet-ring cells gastric adenocarcinoma. The patient was referred for chemotherapy. After a short-term partial remission, he died within two months after the final diagnosis. The presented case illustrates challenges posed by the diagnostic process of pulmonary tumour embolism.

Key words: pulmonary tumour embolism, signet-ring gastric adenocarcinoma, dyspnoea, pleural effusion.

Contemp Oncol (Pozn) 2020; 24 (4): 258-262 DOI: https://doi.org/10.5114/wo.2020.102631

\section{The diagnostic challenge in pulmonary tumour embolism in cancer: a case report and literature review}

\author{
Aleksandra Zielińska ${ }^{1}$, Marcin Braun ${ }^{1}$, Dominika Piasecka ${ }^{1}$, Piotr Minc ${ }^{2}$, \\ Hanna M. Romańska ${ }^{1}$
}

1'Department of Pathology, Chair of Oncology, Medical University of Lodz, Lodz, Poland 2Department of Internal Diseases, Copernicus Memorial Hospital, Lodz, Poland

\section{Introduction}

Pulmonary tumour embolism (PTE) is a rare condition characterised by obstruction made up of tumour cells and thrombi in the pulmonary arterioles and capillaries, diagnosis of which antemortem is notoriously difficult. Clinically it is manifested by nonspecific symptoms such as dyspnoea, cough and pulmonary hypertension [1]. The majority of routine diagnostic procedures fail to accurately identify PTE. Moreover, PTE can occur as the first manifestation of cancer and, as such, mistaken for a cardiopulmonary pathology, it may cause a delay in diagnosis of the underlying malignancy.

Here we report a case of a 46-year-old Caucasian male with multiple pulmonary tumour embolism derived from hitherto undiagnosed malignant disease. The presented case demonstrates the ambiguities surrounding the diagnosis of PTE.

\section{Case report}

In 2018, a 46-year-old male was admitted to the Department of Internal Medicine with a one-month history of progressing dyspnoea at rest. The patient reported cough with mucus and severe exercise intolerance. In the last year he had weight loss of 15 kilograms. The patient had no past history of either pulmonary or heart problems. He was a heavy smoker for 17 years and a stonemason by profession. Because of an occupational hazard, pneumoconiosis was initially suspected.

On examination the patient was tachycardiac with normal blood pressure (120/78 mm Hg), tachypnoeic and hypoxic ( $\mathrm{SpO}_{2} 93.7 \%$ in room air). Mild exercise induced oxygen desaturation. Laboratory tests indicated acute inflammation. Spirometry demonstrated mild lung restriction. Bronchoscopy did not reveal any relevant anomalies. Microbiological analysis of the aspirate provided negative results. No significant abnormalities were detected by abdominal ultrasound, electrocardiography or echocardiography. Pulmonary angiography disclosed no evidence of emboli. X-ray imaging showed several pathologic features such as fluid in pleural space, loss of pulmonary lobes volume and reticular opacification (Fig. 1). High-resolution computed tomography (HRCT) (Fig. 2) displayed left lung base atelectasis, interlobular septal thickening and pleural effusion (left space $-65 \mathrm{ml}$, right space $-20 \mathrm{ml}$ ). The HRCT also detected an elevation of the left hemidiaphragm and mediastinal nodes enlargement. Subsequently, thoracentesis was performed to aspirate exudative fluid from the pleural cavity $(2650 \mathrm{ml})$. The patient received antibiotics and oxygen therapy, but no improvement was observed. Based on the clinical presentation as well as on the CT scan, the differential diagnosis included sarcoidosis, silicosis and lymphangitic carcinomatosis. In search for a specific causal factor the patient was further subjected to video-assisted 


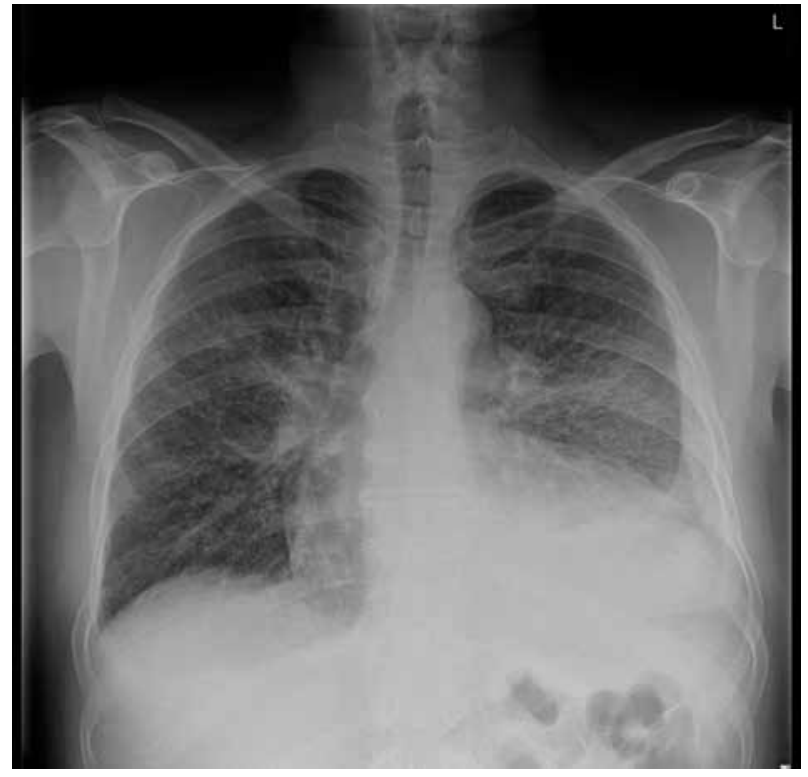

Fig. 1. X-ray imaging showing fluid in pleural space, loss of pulmonary lobes volume and reticular opacification

thoracoscopic surgery (VATS), during which pleurodesis was performed and biopsies of both parietal pleura and lung parenchyma were taken.

Results of histopathological examination excluded all initially suggested underlying conditions. On high magnification, multiple emboli of atypical epithelial cells were found in pulmonary blood vessels (Fig. 3A). The cells had a characteristic signet - ring shape with a central, optically clear droplet of cytoplasmic mucin and eccentrically displaced nucleus (Figs. 3B and 3C). Immunohistochemistry (IHC) revealed i) positivity for cytokeratin 7, cytokeratin 20, cytokeratin 19, Ki67, high molecular weight cytokeratin and cytokeratin AE1/AE3 (Fig. 4) and ii) negativity for CD68, thyroid transcription factor 1 (TTF1), cytokeratin 5/6, p63, synaptophysin, CD10, napsin A, PAX-8, prostate specific membrane antigen (PSMA), adult renal cell carcinoma (RCC), CD56 and chromogranin A. Results for CDX2 were heterogenous (Table 1). In addition, the biopsy was found positive for mucin.

Since the IHC data strongly suggested the gastrointestinal (GI) tract as the origin of cells, Gl endoscopy was conducted. Inflammatory changes within the gastric mucosa were observed. Two ulcers covered with fibrin measuring approximately $6 \mathrm{~mm}$ and $10 \mathrm{~mm}$ in diameter were detected in the stomach. Biopsy was taken and histopathology confirmed the diagnosis of signet ring cell gastric adenocarcinoma (Fig. 5) with haematogenous dissemination involving pulmonary vessels. The patient was referred for palliative EOX (epirubicin, oxaliplatin, capecitabine) chemotherapy. After completing two treatment cycles he reached partial remission and stabilisation of the disease. One month later his condition deteriorated and because of exacerbation of dyspnoea, he required an oxygen concentrator. He received four cycles of docetaxel, but pleural effusion was constantly increasing. The patient underwent several thoracenteses. There was no improvement and he died within two months from the first chemotherapy.
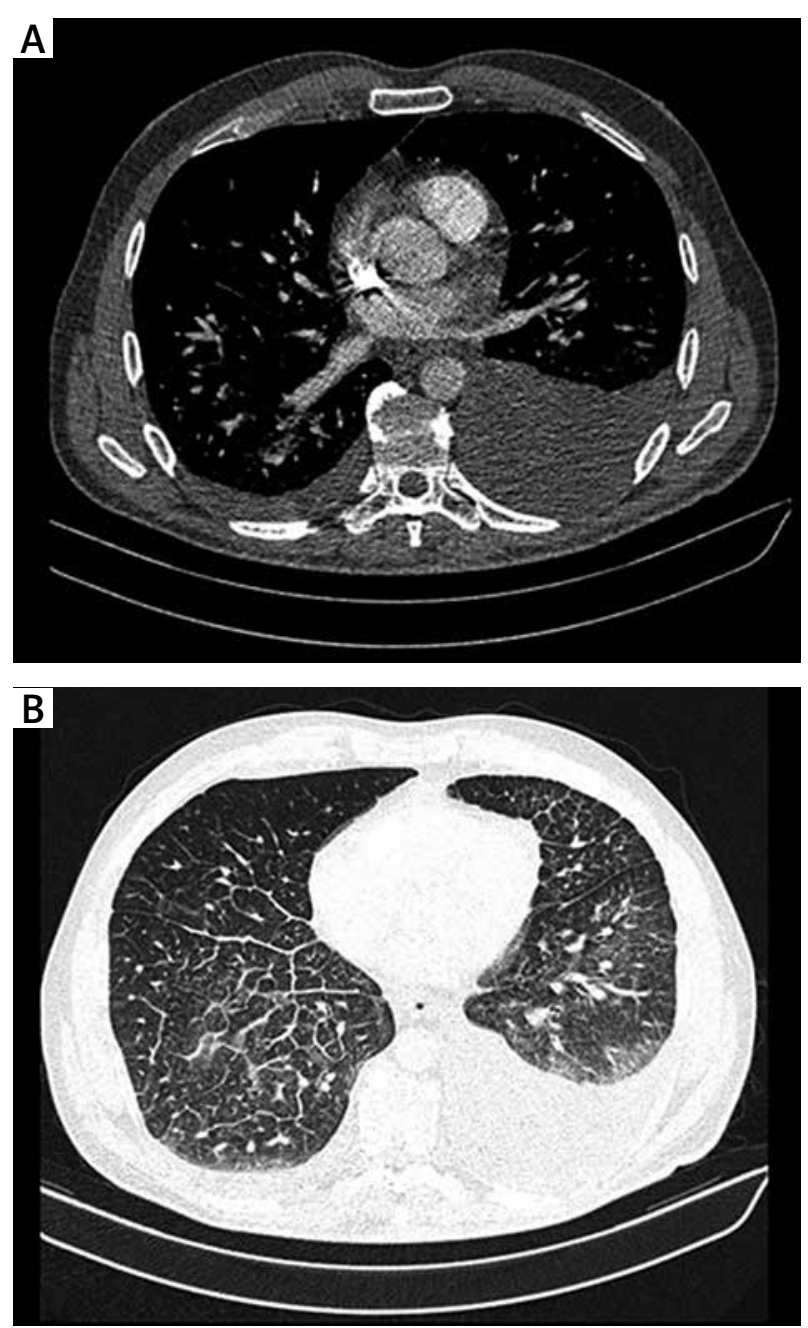

Fig. 2. High-resolution computed tomography (HRCT): fluid in pleural cavity (A), interlobular septal thickening, mediastinal nodes enlargement (B)

\section{Discussion}

We described a case of PTE presented with dyspnoea that was associated with previously unrecognized signet ring cell gastric adenocarcinoma, diagnosis of which created considerable difficulties.

PTE is characterized by a presence of isolated cells or clusters of tumour cells within the pulmonary arterioles causing microthrombi and occlusion, which leads to the elevation of pulmonary vascular resistance and development of hypertension (PH) [2]. The condition was documented for the first time by Schmidt in 1897 [3]. In the 2004 World Health Organization (WHO) Classification, PTE belongs to the "group 4" of the Clinical Category of Pulmonary Hypertension [4]. The most recent WHO Classification's update (2016) assigned PTE to the "Other malignant tumours" group $(4.2 .2)[5,6]$. PTE can evolve into concomitant pulmonary tumour thrombotic microangiopathy (PTTP), characterised by fibrocellular proliferation of arterial intima [7].

PTE is rarely reported in cancer patients, which is mainly due to the considerable difficulty of antemortem diagnosis. It is assumed that microscopic PTEs are found posthumously in approximately $3 \%$ to $26 \%$ of solid malignant neoplasms 

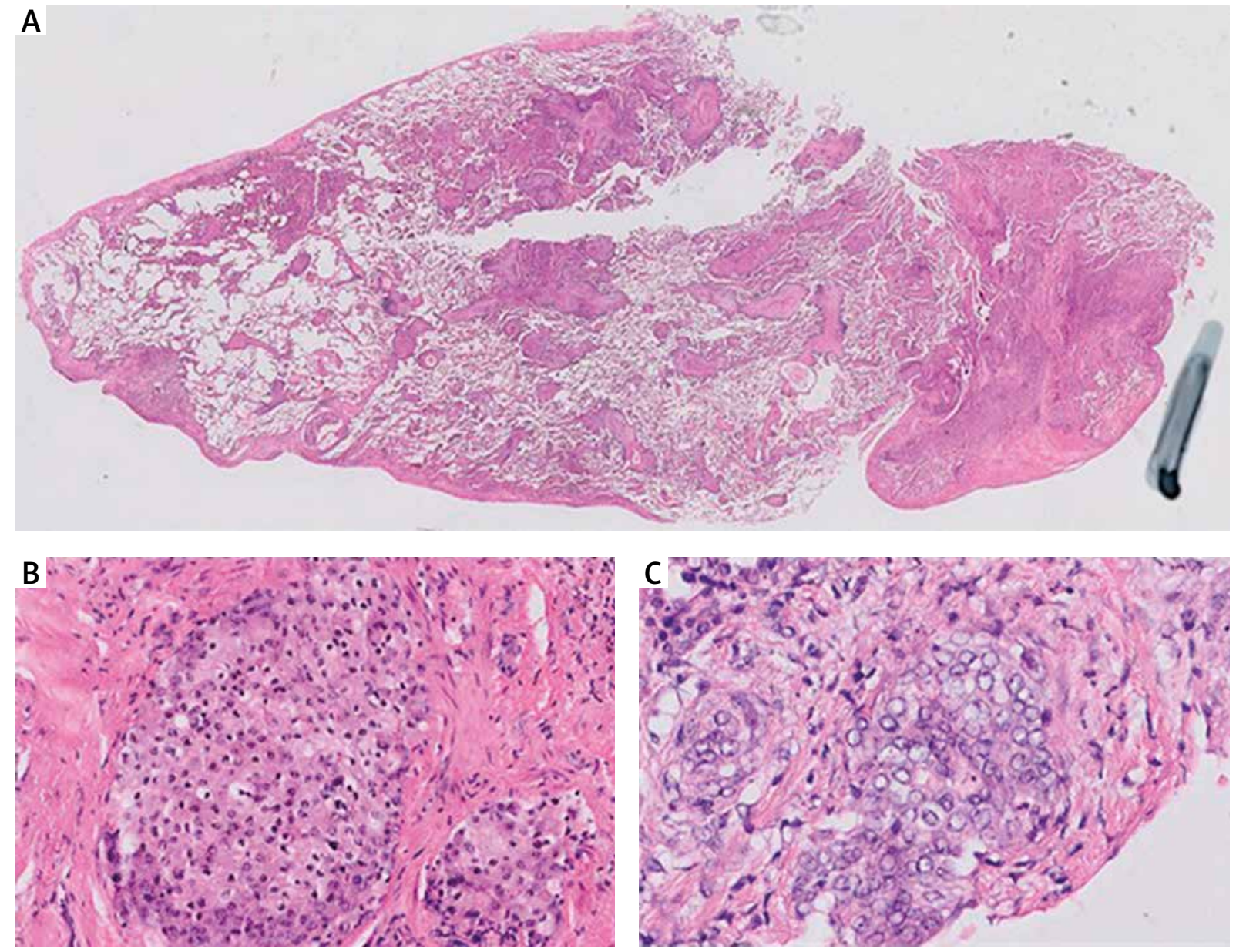

Fig. 3. The haematoxylin and eosin staining shows multiple emboli of atypical epithelial cells (A, B). The cells have a characteristic signet - ring shape with a central, optically clear droplet of cytoplasmic mucin and eccentrically displaced nucleus (C). Magnification of $0.5 \times$ (A), 20× (B), 40× (C)
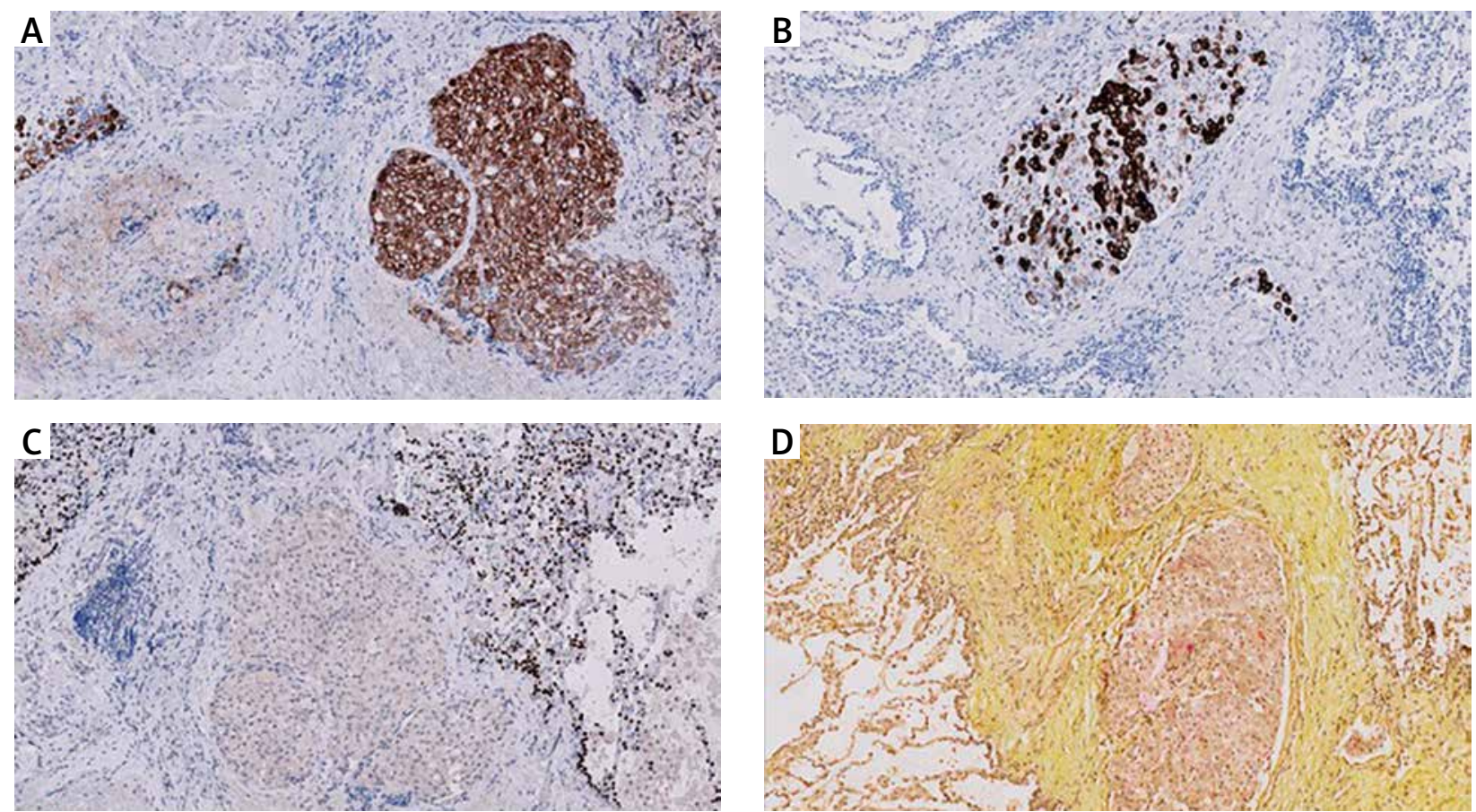

Fig. 4. Immunophenotyping of tumour cells: positivity for cytokeratin 7 (A), cytokeratin 20 (B), negativity for TTF1 (C). The biopsy was positive for mucin (D). Magnification of 10x 
Table 1. List of primary antibodies used in the immunohistochemistry panel

\begin{tabular}{|c|c|c|c|}
\hline Antibody & Clone & Vendor & CAT \# \\
\hline Cytokeratin 7 & OV-TL $12 / 30$ & Dako & IR619 \\
\hline Cytokeratin 20 & Ks20.8 & Dako & IR777 \\
\hline Cytokeratin 19 & RCK108 & Dako & IR615 \\
\hline Ki 67 & MIB-1 & Dako & IR626 \\
\hline $\begin{array}{l}\text { High molecular weight } \\
\text { cytokeratin }\end{array}$ & $34 \beta E 12$ & Dako & |R051 \\
\hline Cytokeratin AE1/AE3 & AE1/AE3 & Dako & IR053 \\
\hline CD68 & KP1 & Dako & IR609 \\
\hline TTF1 & $8 \mathrm{G} 7 \mathrm{G} 3 / 1$ & Dako & IR056 \\
\hline Cytokeratin 5/6 & D5/16 B4 & Dako & IR780 \\
\hline p63 & DAK-p63 & Dako & M7317 \\
\hline Synaptophysin & SY38 & Dako & IR776 \\
\hline CD10 & $56 C 6$ & Dako & IR648 \\
\hline Napsin A & MRQ-60 & Roche & $760-4867$ \\
\hline PAX-8 & MRQ-50 & Roche & $760-4618$ \\
\hline PSMA & $3 \mathrm{E} 6$ & Dako & M3620 \\
\hline RCC & SPM314 & Dako & IR075 \\
\hline CD56 & $123 \mathrm{C} 3$ & Dako & IR628 \\
\hline Chromogranin A & DAK-A3 & Dako & IR502 \\
\hline CDX2 & DAK-CDX2 & Dako & M3636 \\
\hline
\end{tabular}

that do not have metastases to the lung parenchyma $[2,8]$. Since the condition is exceptionally infrequent, the patient might be misdiagnosed and, as a consequence, treated for more common diseases. In fact, making a diagnosis of PTE, like in our case, while the patient was still alive, is uncommon. The most of PTE cases originate from adenocarcinomas of the breast, stomach, lung, colon and prostate [9]. There are few reports of PTE associated with choriocarcinoma [10, 11], pelvic chondrosarcoma [12] myxofibrosarcoma [13] and renal sarcoma [14]. Diagnosis of PTE is usually made coincidentally at postmortem examination [15-17]. It is assumed that PTE may be the cause of death in $3.6 \%$ of cancer patients [18].

Clinical manifestation of PTE is uncharacteristic. The condition can present in a wide range of nonspecific symptoms, sometimes very similar to those of other more common cardiopulmonary diseases. Progressive dyspnoea is the main clinical manifestation of PTE (present in 57\% up to $100 \%$ cases) [2]. Shortness of breath results in arterial hypoxia and respiratory alkalosis. Cough and chest pain commonly accompany PTE (47\% of cases) and $15-20 \%$ of PTE patients demonstrate classic signs of pulmonary hypertension [19] likely to be associated with right heart failure (pulmonary heart disease) $[17,20,21]$. Except for progressive dyspnoea, the above symptoms did not contribute to the clinical presentation of the reported case. Instead of pulmonary hypertension and right heart failure, the patient suffered from tachypnoea and tachycardia. A few symptoms characteristic of PTE such as cough, dyspnoea and mild lung restriction the patient complained of were misinterpreted as silicosis, a condition more common and, because of the

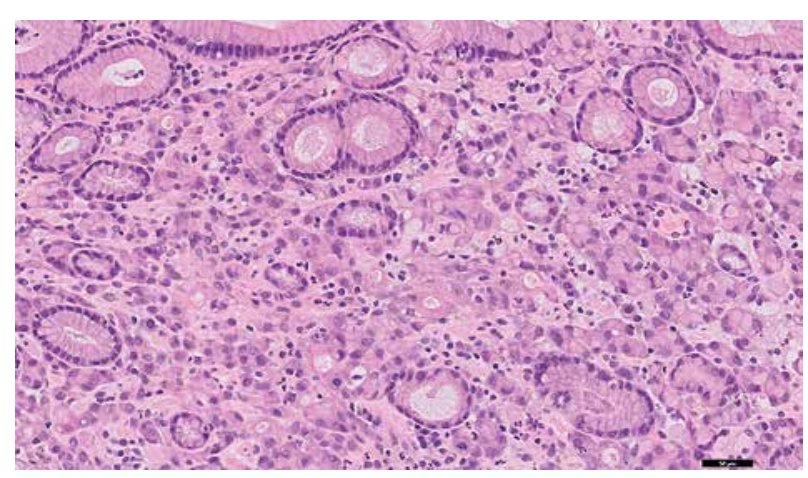

Fig. 5. The haematoxylin and eosin staining of signet ring cell gastric adenocarcinoma. Magnification of $20 x$

patient's occupational hazard (a stonemason) and cigarette smoking, deemed more likely to be true. Results of the initial examination could also suggest sarcoidosis or lymphangitic carcinomatosis, as the patient suffered from massive pleural effusion, a symptom reported in some silicosis [22], lymphangitic carcinomatosis [23], and sarcoidosis [24] but never in PTE cases.

PTE has typically a poor prognosis. In the majority of cases patients die shortly after admission due to cardiopulmonary arrest. Unfortunately, there are no treatment guidelines available - only a few treatment options have been recommended. Anticoagulants were widely proved not to increase the survival rate. As reported in some cases, early chemotherapy or surgical embolectomy of pulmonary embolism followed by chemotherapy might be successful and lead to prolonged survival [10, 12, 25-27].

Early recognition of PTE would be essential, but it is difficult before death since the range of diagnostic procedures described over the years is very limited. Surgical lung biopsy with histopathological evaluation is the gold standard and indeed, in the presented case, it played a key role in establishing the diagnosis. Microscopically, the pulmonary vessels are occluded by emboli consisting of atypical malignant cells. Typically, there is no evidence of adjacent primary or metastatic tumours. A wide panel of immunohistochemical staining is highly recommended for verification of possible cell origin. In our case, the cells of gastric adenocarcinoma were found within pulmonary vessels and the extrapulmonary origin of cells was confirmed by performing specific immunohistochemistry. Concerning hemodynamically unstable patient, transbronchial biopsy is considered to be an alternative diagnostic test, but it must be avoided in patients with severe $\mathrm{PH}$ due to the high risk of bleeding [26]. Radiographic examination usually shows a normal chest X-ray with associated hypoxia and is not diagnostic in PTE [27]. Parenchymal abnormalities (atelectasis, reticular pattern) in chest radiography, as seen in the presented case, are rare [17]. Moreover, they are characteristic of other more common conditions, and did not assist proper assessment and diagnosis. Pulmonary angiography typically fails to identify PTE [17, 21], although in a few cases it was reported to guide the diagnosis [10]. HRCT is often normal, therefore as a diagnostic tool it also remains of limited value [26]. There are some reports about the utility of a ventilation-perfusion scintigraphy, which usually reveals 
numerous, symmetrical, peripherally located, subsegmental perfusion defects ("mottled" or "beaded" appearance) with correct ventilation $[26,29,30]$. Pulmonary microvascular cytology (PMC) has been proposed as an additional test, however its sensitivity and specificity are doubtful $[27,28]$. Therefore, in the majority of cases the actual diagnosis of PTE is made at autopsy.

\section{Conclusions}

PTE is a rare entity with a nonspecific clinical presentation and can cause diagnostic difficulties. As it may be the initial manifestation of malignancy, it is essential to always consider PTE in the differential diagnosis of progressive dyspnoea.

The authors declare no conflict of interest.

\section{References}

1. Roberts KE, Hamele-Bena D, Saqi A, Stein CA, Cole RP. Pulmonary tumor embolism: A review of the literature. Am J Med 2003; 115: 228-232.

2. Price LC, Seckl MJ, Dorfmüller P, Wort SJ. Tumoral pulmonary hypertension. Eur Respir Rev 2019; 28: 180065.

3. Schmidt MB. Die Verbreitungswege der Karzinome und die Beziehung generalisierter Sarcome zu den leukaemischen Neubildungen. G. Fischer, Vienna 1903.

4. Simonneau G, Galiè N, Rubin LJ, et al. Clinical classification of pulmonary hypertension. J Am Coll Cardiol 2004; 43 (Suppl): 5S-12S.

5. Simonneau G, Montani D, Celermajer DS, et al. Haemodynamic definitions and updated clinical classification of pulmonary hypertension. Eur Respir J 2019; 53: 1801913.

6. Yaghi S, Novikov A, Trandafirescu T. Clinical update on pulmonary hypertension. J Investig Med 2020; 68: 821-827.

7. Uruga H, Fujii T, Kurosaki A, et al. Pulmonary tumor thrombotic microangiopathy: A clinical analysis of 30 autopsy cases. Intern Med 2013; 52: 1317-1323.

8. Kane RD, Hawkins HK, Miller JA, Noce PS. Microscopic pulmonary tumor emboli associated with dyspnea. Cancer 1975; 36: 14731482.

9. Sakuma M, Fukui S, Nakamura M, et al. Cancer and pulmonary embolism - thrombotic embolism, tumor embolism, and tumor invasion into a large vein. Circ J 2006; 70: 744-749.

10. Chung JH, Yeo HJ, Cho HM et al. Treatment of pulmonary tumor embolism from choriocarcinoma: extracorporeal membrane oxygenation as a bridge through chemotherapy. Cancer Res Treat 2017; 49: 279-282.

11. Chai L, Ong KC, Ng SB. A case of pulmonary tumour embolism mimicking miliary tuberculosis. Respirology 2000; 5: 297-299.

12. Chandrasekharan R, Bhagavaldas MC, Mathew AJ. Chondrosarcoma presenting as dyspnea in a 19-year-old man: A case report. J Med Case Rep 2011; 5:150

13. Latchana N, Daniel VC, Gould RW, Pollock RE. Pulmonary tumor embolism secondary to soft tissue and bone sarcomas: A case report and literature review. World I Surg Oncol 2017; 15: 1-7.

14. Yang JH, Song DH, Lee C, et al. Malignant pulmonary embolism associated with renal sarcoma: A case report. Medicine (Baltimore) 2020; 99: 1-5.

15. Chandler C. Malignant arterial tumor embolization. J Surg Oncol 1993; 52: 197-202.

16. Heithaus RE, Hitchcock MA, Guileyardo JM. Pulmonary tumor embolism syndrome from occult colonic adenocarcinoma. Bayl Univ Med Cent Proc 2013; 26: 290-292.

17. Schriner RW, Ryu JH, Edwards WD. Microscopic pulmonary tumor embolism causing subacute cor pulmonale: a difficult antemortem diagnosis. Mayo Clin Proc 1991; 66: 143-148.
18. Winterbauer RH, Elfenbein IB, Ball WC. Incidence and clinical significance of tumor embolization to the lungs. Am J Med 1968; 45: 271-290.

19. Bassiri AG, Haghighi B, Doyle RL, Berry GJ, Rizk NW. Clinical commentary pulmonary tumor embolism. Am J Respir Crit Care Med 1997; 155: 2089-2095

20. Iwakami SI, Sato T, Takagi H, et al. An autopsy case of subacute cor pulmonale due to pulmonary tumor cell emboli in a patient with gastric cancer. Intern Med 2009; 48: 1057-1060.

21. Nakamura $H$, Adachi $H$, Sudoh $A$, et al. Subacute cor pulmonale due to tumor embolism. Intern Med 2004; 43: 420-422.

22. Rout P, Markam G. Pleural effusion in silicosis: A rare case report. Med I DY Patil Vidyapeeth 2018; 11: 436-438.

23. Garcia Sevila R, Barroso E, Martin C, Aranda I, Romero S. Lymphangitic carcinomatosis as a cause of malignant transient pleural transudate. Case Rep Med 2009; 2009: 1-3.

24. Joshi S, Periwal P, Dogra V, Talwar D. Sarcoidosis as unusual cause of massive pleural effusion. Respir. Med. Case Reports 2015; 16: 143-145.

25. Chung JH, Yeo HJ, Cho HM, et al. Treatment of Pulmonary Tumor Embolism from Choriocarcinoma: Extracorporeal Membrane Oxygenation as a Bridge through Chemotherapy. Cancer Res Treat 2017; 49: 279-282.

26. Ishiguro T, Takayanagi N, Baba Y, Kagiyama N, Miyamoto T, Mutoh M, Shimizu Y, Sugita Y. Case series of pulmonary tumor embolism and intravascular lymphoma: evaluation of the usefulness of pulmonary microvascular cytology. Intern Med 2016; 55: 2679-2684.

27. Morin-Thibault LV, Wiseman D, Fortin M, Couture C, Provencher S. Pulmonary micro-tumor emboli resulting in paradoxical emboli: a case report. Pulm Circ 2018; 8: 2045893218754853.

28. Abati A, Landucci D, Danner RL, Solomon D. Diagnosis of pulmonary microvascular metastases by cytologic evaluation of pulmonary artery catheter-derived blood specimens. Hum Pathol 1994; 25: 257-262.

29. Chen WL, Cherng SC, Hwang WS, Wang DJ, Wei J. Perfusion scan in pulmonary tumor microembolism: report of a case. J Formos Med Assoc 1991; 90: 863-866.

30. Crane R, Rudd TG, Dail D. Tumor microembolism: pulmonary perfusion pattern. J Nucl Med 1984; 25: 877-880.

\section{Address for correspondence}

\section{Marcin Braun}

Department of Pathology

Chair of Oncology

Medical University of Lodz

251 Pomorska St.

92-213 Lodz, Poland

e-mail: braunmarcin@gmail.com

Submitted: 28.06 .2020

Accepted: 15.07 .2020 